\title{
DIHEDRAL SEMIGROUPS, THEIR DEFINING RELATIONS AND AN APPLICATION TO DESCRIBING KNOT SEMIGROUPS OF RATIONAL LINKS
}

\author{
ALEXEI VERNITSKI, LAILA TUNSI, COLETTE PONCHEL, AND ALEXEI LISITSA
}

\begin{abstract}
It is known that a knot (link) is rational if and only if its $\pi$-orbifold group is dihedral. A semigroup version of this result has been formulated as a conjecture. Working towards proving the conjecture, we describe certain semigroups associated with twist links, clarify how these semigroups are related to dihedral groups and find defining relations of these semigroups.
\end{abstract}

\section{Definitions}

Consider a braid with 4 strands whose ends on the left-hand side and the righthand side are connected in one way or another to form closed curves (see an example on Figure 1.1); we shall refer to such a diagram as a 4-plat diagram. To make this description more formal, enumerate positions of strands of the braid from the bottom to the top as $1,2,3,4$. Denote the clockwise half-twist of strands in positions 1 and 2 (or 2 and 3 , or 3 and 4 ) by $\sigma_{1}$ (or $\sigma_{2}$, or $\sigma_{3}$ ). Thus, for instance, the braid part of the 4-plat diagram on Figure 1.1, if we inspect it from the left to the right, is $\sigma_{1}^{-1} \sigma_{2} \sigma_{3}$. On the left-hand side of a 4-plat diagram either the strands in positions 1 and 2 and the strands in positions 3 and 4 are connected (we shall refer to this as the adjacent connection), or, alternatively, the strands in positions 1 and 4 and the strands in positions 2 and 3 are connected (we shall refer to this as the nested connection). Likewise, ends of strands are closed in either of these two ways on the right-hand side of a 4-plat diagram.

Knots (links) represented by 4-plat diagrams are called 4-plats or rational knots (links) or 2-bridge knots (links).

Given a diagram of a knot (or a link), one can define a number of algebraic constructions, including groups, quandles and keis, which reflect properties of the diagram or of the knot represented on the diagram. In [10] a knot semigroup was defined as follows. By an arc we mean a continuous line on a knot diagram from one undercrossing to another undercrossing. To denote a crossing on a knot diagram

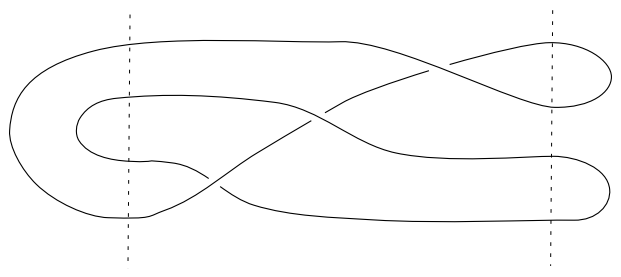

FiguRE 1.1. An example of a 4-plat diagram. 

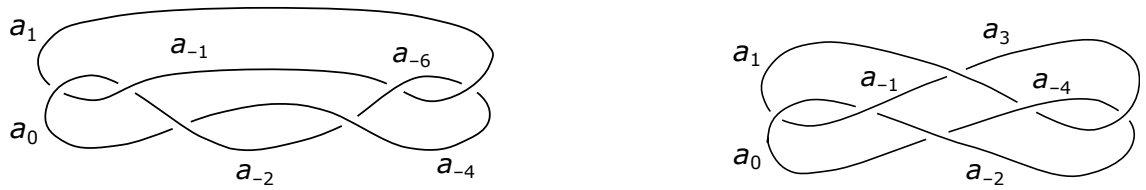

FiguRE 1.2. Twist link diagrams $\mathfrak{t w}_{2}^{0}$ and $\mathfrak{t w}_{1}^{1}$

we shall use notation $x \dashv y \vdash z$, where $x$ and $z$ are the two arcs terminating at the crossing and $y$ is the arc passing over the crossing. To define the knot semigroup of a diagram $\mathfrak{d}$, assume that each arc is denoted by a letter. Then at every crossing $x \dashv y \vdash z$, 'read' two defining relations $x y=y z$ and $y x=z y$. (For instance, the leftmost crossing of either diagram on Figure 1.2 produces two defining relations $a_{1} a_{0}=a_{0} a_{-1}$ and $a_{0} a_{1}=a_{-1} a_{0}$.) The cancellative semigroup generated by the arc letters with these defining relations is the knot semigroup $K \mathfrak{d}$ of $\mathfrak{d}$. The purpose of this paper is to present our progress in describing knot semigroups of 4-plat diagrams.

The standard diagram of a torus knot (if $n$ is odd) or a torus link (if $n$ is even) $T(2, n)$ looks like a 4-plat diagram whose braid part is $\sigma_{1}^{n}$ for some non-negative integer $n$, with the nested connection at both ends ${ }^{1}$. The standard diagram of a twist knot with $n+2$ crossings, where $n$ is a non-negative integer, looks like a 4-plat diagram whose braid part is $\sigma_{1}^{n} \sigma_{2}^{-2}$, with the nested connection on the left and the adjacent connection on the right. Knot semigroups of standard diagrams of torus knots and torus links $T(2, n)$ and of standard diagrams of twist knots are described in [10].

In this paper we consider a slightly more complicated construction of twist links; for our purposes, a standard diagram of twist links with $c+d+4$ crossings, where $c$ and $d$ are non-negative integers, is a 4-plat diagram $\mathfrak{t w}_{c}^{d}$ with the braid part $\sigma_{2}^{-2} \sigma_{1}^{c} \sigma_{3}^{d} \sigma_{2}^{-2}$ or, equivalently, $\sigma_{2}^{-2} \sigma_{3}^{d} \sigma_{1}^{c} \sigma_{2}^{-2}$, and the adjacent connection at both ends. For example, $\mathfrak{t w}_{2}^{0}$ and $\mathfrak{t w}_{1}^{1}$ are shown on Figure 1.2.

Let $B \subseteq \mathbb{Z}_{n}$ for some fixed positive integer $n$. By the alternating sum alt $(u)$ of a word $u=b_{1} b_{2} b_{3} b_{4} \ldots b_{k} \in B^{+}$we shall mean the value of the expression $b_{1}-b_{2}+b_{3}-b_{4}+\cdots+(-1)^{k+1} b_{k}$ calculated in $\mathbb{Z}_{n}$. We shall say that two words $u, v \in B^{+}$are in relation $\sim$ if and only if $u$ and $v$ have the same length and $\operatorname{alt}(u)=\operatorname{alt}(v)$. It is obvious that $\sim$ is a congruence on $B^{+}$. We denote the factor semigroup $B^{+} / \sim$ by $A S_{n}(B)$ and call it an alternating sum semigroup [10]. In Section 4 we shall see why one can also call alternating sum semigroups dihedral semigroups. Now suppose that $n$ is even. We shall say that two words $u, v \in B^{+}$ are in relation $\approx$ if and only if $u$ and $v$ are in relation $\sim$ and the number of odd entries in $u$ is equal to the number of odd entries in $v$ (or, equivalently, the number of even entries in $u$ is equal to the number of even entries in $v$ ). It is obvious that $\approx$ is a congruence on $B^{+}$. We denote the factor semigroup $B^{+} / \approx$ by $S A S_{n}(B)$ and call it a special alternating sum semigroup [10].

The factor semigroup of $K \mathfrak{d}$ relative to the congruence induced by equalities $x x=1$ for each arc $x$ is a group known to knot theorists as the $\pi$-orbifold group

\footnotetext{
${ }^{1}$ Some minor variations of the constructions are possible, which do not affect the knot semigroups. For instance, the torus knot (link) can be considered as one represented by a 4-plat diagram whose braid part is $\sigma_{1}^{-n}$ instead of $\sigma_{1}^{n}$, as discussed in the beginning of Section 4 in [10].
} 
$O \mathfrak{d}$. The following result is relevant to our $\mathrm{study}^{2}$; it is first proved as Proposition 3.2 in [1], or see the survey [7]. We present an elegant algebraic proof of the 'only if' part of this result in Section 5.

Fact 1. A knot or a link is a 4-plat if and only if its $\pi$-orbifold group is dihedral.

The following conjecture was formulated in [10]; we still cannot prove it. The conjecture seems plausible for several reasons. First, the conjecture is true for some partial cases: torus knots $T(2, n)$ and twist knots [10]. Second, Fact 1 is consistent with this conjecture (see the discussion in the end of Section 4). Third, to test this conjecture, knot semigroups of all 4-plat knot diagrams with up to 9 crossings were described using computer [8].

Conjecture 2. The knot semigroup of a 4-plat knot diagram is isomorphic to an alternating sum semigroup.

The following new conjecture can be formulated for links. The conjecture is true for torus links $T(2, n)[10]$. Fact 1 seems consistent with this conjecture. A weaker Conjecture 4 (below) is true for twist links, as we prove in this paper.

Conjecture 3. The knot semigroup of a 4-plat link diagram is isomorphic to a special alternating sum semigroup.

While we cannot prove Conjecture 3 , we formulate a weaker conjecture as follows. By a component semigroup we mean a subsemigroup of a knot semigroup of a link diagram generated by arcs belonging to one component of the link.

Conjecture 4. Each component semigroup of a 4-plat link diagram is isomorphic to an alternating sum semigroup.

The central result of this paper is a theorem proving Conjecture 4 for twist links.

Let us explain briefly why the structure of knot semigroups of 4-plat knot diagrams (Conjecture 2) can be expected to be so different from that of 4-plat link diagrams (Conjecture 3), unlike the unified picture for groups in Fact 1 . One possible explanation is that relations of knot semigroups allow us to rewrite words in a way which preserves the number of letters from each link component (to see how this property can be used, see Example 14). Thus, the separate treatment of odd letters and even letters in a special alternating sum semigroup is a convenient way of expressing the fact that in a 4-plat link diagram we have arcs of two sorts, that is, those belonging to one component and those belonging to the other component.

\section{Presentations for $A S_{n}(B)$}

In [10] we proved that a knot semigroup $K \mathfrak{d}$ is isomorphic to an alternating sum semigroup $A S_{n}(B)=B^{+} / \sim$ by selecting, with some difficulty, a canonical word in each class of $\sim$ and then proving that there is a one-to-one correspondence between the elements of $K \mathfrak{d}$ and the canonical words in $B^{+}$. In this paper we use an easier approach, which enables us to progress to considering more complicated links. We proved in [8] that each alternating sum semigroup has a finite presentation. If we want to prove that $K \mathfrak{d}$ (or a certain subsemigroup of $K \mathfrak{d}$ ) is isomorphic to a certain

\footnotetext{
${ }^{2}$ We are grateful to José Montesinos (Universidad Complutense de Madrid), Genevieve Walsh (Tufts University) and Vanni Noferini (University of Essex) for attracting our attention to this result.
} 
$A S_{n}(B)$ and we have a presentation for $A S_{n}(B)$, we only need to prove that every defining relation of $A S_{n}(B)$ follows from the defining relations of $K \mathfrak{d}$. In Theorem 5 of this section we find presentations for some alternating sum semigroups (doing this, we still consider words of a canonical form, which we denote by $w(m, i)$, but we do not have to match them to classes of $\sim$ ). Then in Section 3 we use these presentations to prove that these alternating sum semigroups are isomorphic to component semigroups of twist links.

Theorem 5. $A S_{2 n}(\{0, \ldots, k-1\})$ is defined, as a cancellative semigroup, by relations

(1) $00=11$;

(2) $a 00=(a-1) 01$ for $a=1, \ldots, k-1$;

(3) $a 10=(a-1) 11$ for $a=1, \ldots, k-1$;

(4) $(01)^{n}=(10)^{n}$.

For a word $w$ and a non-negative integer $i$, notation $w^{i}$ (like in relation (4) above) stands for $w$ repeated $i$ times. For any $i \in 0, \ldots, 2 n-1$ and any sufficiently large positive integer $m$ introduce words $w(m, i)$ as $(10)^{i} 0^{m-2 i}$.

Lemma 6. If a cancellative semigroup satisfies relations (1)-(4) then

1) every palyndrome word of an even length $2 p$ consisting of $0 s$ and $1 s$ is equal to $0^{2 p}$;

2) $(10)^{2 n}=(00)^{2 n}$.

3) $(10)^{i}(00)^{2 n-i}=(01)^{2 n-i}(00)^{i}$ for any $i \in 0, \ldots, 2 n-1$.

4) $0 w(m, i)=w(m+1,2 n-i)$ for any $i \in 0, \ldots, 2 n-1$ and for any sufficiently large $m$.

Proof. 1) We shall use induction on $p$. If $p=0$, the word is empty and is equal to $0^{2 p}$. Now consider a palyndrome word $u$ of length $2 p$. We have either $u=0 v 0=$ $00^{2 p-2} 0=0^{2 p}$ or $u=1 v 1=10^{2 p-2} 1=0^{2 p-2} 11=0^{2 p-2} 00=0^{2 p}$.

2) Using the result of part 1, we have $(10)^{2 n}=(10)^{n}(10)^{n}=(01)^{n}(10)^{n}=(00)^{2 n}$.

3) We have $(10)^{i}(01)^{i}(00)^{2 n-i}=(00)^{2 n+i}=(10)^{i}(10)^{2 n-i}(00)^{i}$. Using cancellation, we conclude $(10)^{i}(00)^{2 n-i}=(01)^{2 n-i}(00)^{i}$.

4) We have $w(m, i)=(10)^{i}(00)^{2 n-i} 0^{m-2 n}=(01)^{2 n-i}(00)^{i} 0^{m-2 n}$. Hence, $0 w(m, i)=$ $0(01)^{2 n-i}(00)^{i} 0^{m-2 n}$. By repeatedly using relation (2) to replace 001 by 100 , this word can be rewritten as $(10)^{2 n-i} 0(00)^{i} 0^{m-2 n}$, which is $w(m+1,2 n-i)$.

Lemma 7. If a cancellative semigroup satisfies relations (1)-(4) then aw $(m, i)=$ $w(m+1, a-i)$, where $a-i$ is calculated in $\mathbb{Z}_{2 n}$, for any $a, i \in \mathbb{Z}_{2 n}$ and for any sufficiently large $m$.

Proof. First prove that if $a>0$ then $a w(m, i)=(a-1) w(m, i-1)$, where $i-1$ is calculated in $\mathbb{Z}_{2 n}$. Suppose $i>0$. We have $a w(m, i)=a(10)^{i} 0^{m-2 i}$. Applying relation (3) repeatedly, we obtain $a(10)^{i} 0^{m-2 i}=(a-1)(10)^{i-1}(11) 0^{m-2 i}$. By relation $(1),(a-1)(10)^{i-1}(11) 0^{m-2 i}=(a-1)(10)^{i-1}(00) 0^{m-2 i}=(a-1) w(m, i-1)$. Now suppose that $i=0$. By part 2 of Lemma $6, w(m, 0)=w(m, 2 n)$. Thus, $a w(m, 0)=a w(m, 2 n)=(a-1) w(m, 2 n-1)$.

By applying $a$ times the equality $a w(m, i)=(a-1) w(m, i-1)$, we conclude that $a w(m, i)=0 w(m, i-a)$. By part 4 of Lemma $6,0 w(m, i-a)=w(m+1, a-i)$, where $i-a$ and $i-a$ are calculated in $\mathbb{Z}_{2 n}$.

Proof of Theorem 5. It follows from the definition of $A S_{2 n}(\{0, \ldots, k-1\})$ that relations (1)-(4) are satisfied in $A S_{2 n}(\{0, \ldots, k-1\})$. Now suppose two words 
$u, v \in\{0, \ldots, k-1\}^{+}$are equal in $A S_{2 n}(\{0, \ldots, k-1\})$; then the length of both $u$ and $v$ is the same number $p$, and $\operatorname{alt}(u)=\operatorname{alt}(v)$. Let us prove that the equality $u=v$ can be deduced from relations (1)-(4). Consider words $u w(m, 0)$ and $v w(m, 0)$ for a sufficiently large $m$. Applying Lemma 7 repeatedly, we obtain $u w(m, 0)=$ $w(m+p, \operatorname{alt}(u))$ and $v w(m, 0)=w(m+p, \operatorname{alt}(v))$. Since $\operatorname{alt}(u)=\operatorname{alt}(v), u w(m, 0)=$ $v w(m, 0)$. Hence, $u=v$.

\section{Component SEMigroups of TWist Links}

We index arcs of twist link diagrams with elements of $\mathbb{Z}_{4(c+d)+4}$. For a more convenient notation, we use negative numbers for some indices; this should be interpreted in the natural way: for example, -1 stands for $4(c+d)+3$. In a twist link diagram $\mathfrak{t w}_{c}^{d}$, we denote the arcs of the lower component (from the left to the right) by $a_{0}, a_{-2}, a_{-4}, \ldots, a_{-2 c-2}$ and the arcs of the upper component (from the left to the right, with a slightly confusing order of the first two elements) by $a_{1}, a_{-1}, a_{3}, a_{5}, \ldots, a_{2 d+1}$; see Figure 1.2 for examples. The indices are chosen to match generators of certain alternating sum semigroups, as we shall see in the proof of the theorem.

Theorem 8. 1) The component semigroups of the lower and the upper component of $\mathfrak{t w}_{c}^{d}$ are isomorphic to $A S_{4(c+d)+4}(\{0,-2, \ldots,-2 c-2\})$ and $A S_{4(c+d)+4}(\{-1,1, \ldots, 2 d+$ $1\})$, respectively.

2) The component semigroups of the lower and the upper component of $\mathfrak{t w}_{c}^{d}$ are isomorphic to $A S_{2(c+d)+2}(\{0,1, \ldots, c+1\})$ and $A S_{2(c+d)+2}(\{0,1, \ldots, d+1\})$, respectively.

The isomorphisms in part 1) of the theorem are induced by the mapping $a_{i} \mapsto i$. We can conjecture (but cannot prove) that this mapping also induces an isomorphism between $K \mathfrak{t w}_{c}^{d}$ and $S A S_{4(c+d)+4}(\{0,-2, \ldots,-2 c-2 ;-1,1, \ldots, 2 d+1\})$; this would prove Conjecture 3 for twist links. If we knew defining relations for $S A S_{4(c+d)+4}(\{0,-2, \ldots,-2 c-2 ;-1,1, \ldots, 2 d+1\})$, this would help us to make a progress in describing the knot semigroup of $K \mathrm{tw}_{c}^{d}$, in the same way as defining relations found in Section 2 enable us to prove Theorem 8.

The equivalence of the two parts of the theorem follows from the following observation.

Lemma 9. 1) $A S_{4(c+d)+4}(\{0,-2, \ldots,-2 c-2\})$ is isomorphic to $A S_{2(c+d)+2}(\{0,1, \ldots, c+$ $1\})$;

2) $A S_{4(c+d)+4}(\{-1,1, \ldots, 2 d+1\})$ is isomorphic to $A S_{2(c+d)+2}(\{0,1, \ldots, d+1\})$.

Proof. It is easy to see that $A S_{n}(B)$ is isomorphic to the semigroup $A S_{n}(B+k)$ for any $k \in \mathbb{Z}_{n}$, where $B+k=\{b+k \mid b \in B\}$, and that $A S_{n}(B)$ is isomorphic to the semigroup $A S_{k n}(k B)$, for any positive integer $k$, where $k B=\{k b \mid b \in B\}$ is calculated in $\mathbb{Z}_{k n}$. Hence, $A S_{4(c+d)+4}(\{-1,1, \ldots, 2 d+1\})$ is isomorphic to $A S_{4(c+d)+4}(\{0,2, \ldots, 2 d+2\})$ and, therefore, to $A S_{2(c+d)+2}(\{0,1, \ldots, d+1\})$. Likewise, $A S_{4(c+d)+4}(\{0,-2, \ldots,-2 c-2\})$ is isomorphic to $A S_{4(c+d)+4}(\{0,2, \ldots, 2 c+$ $2\})$ and, therefore, to $A S_{2(c+d)+2}(\{0,1, \ldots, c+1\})$.

Note that by rotating the page half a turn around the axis perpendicular to the page, diagram $\mathfrak{t w}_{c}^{d}$ turns into diagram $\mathfrak{t w}_{d}^{c}$. This is why in this section it is sufficient to prove results only for one ('even-indexed' or 'odd-indexed') half of the diagram; the proofs for the other half are identical. 
Lemmas 10, 11 and 13 below roughly correspond to proving that the relations (1), (2)-(3) and (4) from Theorem 5 are satisfied in the component semigroups of $\mathfrak{t w}_{c}^{d}$.

Lemma 10. 1) $a_{0} a_{0}=a_{-2} a_{-2}$;

2) $a_{-2 c} a_{-2 c}=a_{-2 c-2} a_{-2 c-2}$.

Proof. 1) We have $a_{0} a_{0} a_{-1}=a_{0} a_{1} a_{0}=a_{-1} a_{0} a_{0}=a_{2} a_{-1} a_{0}=a_{2} a_{2} a_{-1}$; the result is then obtained by using cancellation.

2) We have $a_{-2 c} a_{-2 c} a_{2 d+1}=a_{-2 c} a_{2 d+1} a_{-2 c}=a_{2 d+1} a_{-2 c-2} a_{-2 c-2}=a_{-2 c-2} a_{2 d-1} a_{-2 c-2}=$ $a_{-2 c-2} a_{-2 c-2} a_{2 d+1}$; the result is then obtained by using cancellation.

Lemma 11. $a_{i} a_{i+j}=a_{i+k} a_{i+j+k}$ for all possible even values of $i, j$ and $k$.

Proof. Let us prove that $a_{i} a_{i-2}=a_{i+k} a_{i-2+k}$ for all possible even values of $i$ and $k$. Relations in $K \mathrm{tw}_{c}^{d}$ include equalities $a_{i+2} a_{i}=a_{i} a_{i-2}$ for all $i=2,4, \ldots, 2 c$. Applying these relations repeatedly, we obtain $a_{i} a_{i-2}=a_{i+k} a_{i-2+k}$ for all possible even values of $i, k$.

Similarly, by referring to relations of the type $a_{i} a_{i+2}=a_{i-2} a_{i}$, we obtain equalities $a_{i} a_{i+2}=a_{i+k} a_{i+2+k}$ for all possible even values of $i$ and $k$.

Now let us prove that $a_{i} a_{i}=a_{i-4} a_{i-4}$ for all possible values of $i$. We have $a_{i-2} a_{i} a_{i}=a_{i-4} a_{i-2} a_{i}=a_{i-4} a_{i-4} a_{i-2}=a_{i-4} a_{i-6} a_{i-4}=a_{i-2} a_{i-4} a_{i-4}$; by using cancellation, we obtain $a_{i} a_{i}=a_{i-4} a_{i-4}$. By applying these equalities repeatedly, we obtain $a_{i} a_{i}=a_{i+k} a_{i+k}$ for all possible values of $i$ and $k$, where $i$ is even, $k$ is a multiple of 4 , and $i-6 \geq-2 c-2$. Combining these equalities with Lemma 10, we conclude that $a_{i} a_{i}=a_{i+k} a_{i+k}$ for all possible even values of $i$ and $k$.

Now we can prove that $a_{i} a_{i+j}=a_{i+k} a_{i+j+k}$ for all possible even values of $i, j$ and $k$. First assume that $k=2$ and consider $a_{i} a_{i} a_{i+j}=a_{i+j} a_{i+j} a_{i+j}=$ $a_{i+j} a_{i+j+2} a_{i+j+2}=a_{i} a_{i+2} a_{i+j+2}$; hence, $a_{i} a_{i+j}=a_{i+2} a_{i+j+2}$. By applying these equalities repeatedly, we obtain $a_{i} a_{i+j}=a_{i+k} a_{i+j+k}$ for all possible values of $k$.

Corollary 12. 1) $a_{i} a_{0} a_{0}=a_{i+2} a_{0} a_{-2}$ for all possible values of $i$;

2) $a_{i} a_{-2} a_{0}=a_{i+2} a_{-2} a_{-2}$ for all possible values of $i$.

Lemma 13. 1) $a_{i} a_{j}=a_{i+k} a_{j+k}$ for all possible even values of $i$ and $k$ and odd values of $j$;

2) $a_{0} a_{-2 c-2} a_{2 d+1} a_{1}=a_{-2 c-2} a_{0} a_{1} a_{2 d+1}$;

3) $\left(a_{0} a_{-2}\right)^{c+d+1} a_{1} a_{1}=\left(a_{0} a_{0}\right)^{c+d} a_{0} a_{-2 c-2} a_{2 d+1} a_{1}$;

4) $\left(a_{-2} a_{0}\right)^{c+d+1} a_{1} a_{1}=\left(a_{0} a_{0}\right)^{c+d} a_{-2 c-2} a_{0} a_{1} a_{2 d+1}$.

5) $\left(a_{0} a_{-2}\right)^{c+d+1}=\left(a_{-2} a_{0}\right)^{c+d+1}$

Proof. 1) Notice that $a_{i} a_{i} a_{j} a_{j}=a_{-2} a_{-2} a_{-1} a_{-1}=a_{-2} a_{0} a_{1} a_{-1}=a_{i} a_{i+2} a_{j+2} a_{j}$; hence $a_{i} a_{j}=a_{i+2} a_{j+2}$. By applying these equalities repeatedly, we obtain $a_{i} a_{j}=$ $a_{i+k} a_{j+k}$.

2) Using defining relations ${ }^{3}$ and equalities from part 1), we obtain $a_{0} a_{-2 c-2} a_{2 d+1} a_{1}=$ $a_{0} a_{2 d-1} a_{-2 c-2} a_{1}=a_{-2 c-2} a_{2 d-1} a_{0} a_{1}=a_{-2 c-2} a_{-1} a_{0} a_{2 d+1}=a_{-2 c-2} a_{0} a_{1} a_{2 d+1}$.

3 ) and 4) are obtained by repeatedly applying equalities from Lemma 11 and from part 1).

5) follows from parts 2), 3) and 4).

\footnotetext{
${ }^{3}$ For understanding the structure of the proof, it is useful to note that this is the only place where we use relations at both ends of the twist diagram to produce an effect of treating indices as numbers modulo $4(c+d)+4$.
} 
Proof of Theorem 5. Consider a partial mapping $\phi: a_{i} \mapsto i$ from $K \mathfrak{t w}_{c}^{d}$ to $S=$ $S A S_{4(c+d)+4}(\{0,-2, \ldots,-2 c-2 ;-1,1, \ldots, 2 d+1\})$. Since each defining relation of $K \mathrm{tw}_{c}^{d}$ corresponds to a true equality in $S$, mapping $\phi$ can be extended to a homomorphism from $K \mathfrak{t w}_{c}^{d}$ to $S$, which we shall also denote by $\phi$. We shall prove the theorem for the component semigroup $C$ of the lower component of $\mathfrak{t w}_{c}^{d}$, that is, the one consisting of $\operatorname{arcs} a_{0}, a_{-2}, a_{-4}, \ldots, a_{-2 c-2}$. Consider the restriction of $\phi$ to $C$, which we shall denote by $\psi$; then $\psi$ is a homomorphism from $C$ to $S^{\prime}=$ $A S_{4(c+d)+4}(\{0,-2,-4, \ldots,-2 c-2\})$. To prove that $\psi$ is an isomorphism, it is sufficient to prove that the defining relations of $S^{\prime}$ correspond to true equalities in $C$. The defining relations of $S^{\prime}$, which is isomorphic to $A S_{2(c+d)+2}(\{0,1,2, \ldots, c+1\})$, are listed in Theorem 5 , and the corresponding equalities in $C$, which we want to prove, are as follows:

(1) $a_{0} a_{0}=a_{-2} a_{-2}$

(2) $a_{i} a_{0} a_{0}=a_{i+2} a_{0} a_{-2}$ for even $i=-2, \ldots,-2 c-2$;

(3) $a_{i} a_{-2} a_{0}=a_{i+2} a_{-2} a_{-2}$ for even $i=-2, \ldots,-2 c-2$;

(4) $\left(a_{0} a_{-2}\right)^{c+d+1}=\left(a_{-2} a_{0}\right)^{c+d+1}$.

Equality (1) is part 1) of Lemma 10. Equalities (2) and (3) are parts 1) and 2) of Corollary 12. Equality (4) is part 5) of Lemma 13.

Example 14. It is not difficult to notice that the same link is shown on diagrams $\mathfrak{t w}_{2}^{0}$ and $\mathfrak{t w}_{1}^{1}$; for instance, one can transform $\mathfrak{t w}_{2}^{0}$ to $\mathfrak{t w}_{1}^{1}$ by rotating the right half of $\mathfrak{t w}_{2}^{0}$ half a turn clockwise, if one looks from the top of the page ${ }^{4}$. In semigroup $K \mathfrak{t w}_{2}^{0}$ we have $a_{0}^{2}=a_{-2}^{2}=a_{-4}^{2}=a_{-6}^{2}$ and $a_{1}^{2}=a_{-1}^{2}$, by Theorem 8 and because in an alternating sum semigroup the squares of all generators are equal to each other, and $a_{0}^{2} \neq a_{1}^{2}$ because knot semigroup relations preserve the number of letters from each component; therefore, only two words which contain the same number of letters from the lower component and the same number of letter from the upper component can be equal. In semigroup $K \mathfrak{t w}_{2}^{0}$ we have $a_{0}^{2}=a_{-2}^{2}=a_{-4}^{2}$ and $a_{1}^{2}=a_{-1}^{2}=a_{3}^{2}$, and also $a_{0}^{2} \neq a_{1}^{2}$. Thus, both $K \mathfrak{t w}_{2}^{0}$ and $K \mathfrak{t w}_{1}^{1}$ have 6 generators, and the square of each generator can have one of two possible values in each of these semigroups. However, in $K \mathrm{tw}_{2}^{0}$ four generators share one value of the square and two other generators share the other value of the square, whereas in $K \mathfrak{t w}_{1}^{1}$ three generators share one value of the square and three other generators share the other value of the square. Therefore, $K \mathrm{tw}_{2}^{0}$ and $K \mathrm{tw}_{1}^{1}$ are not isomorphic. Thus, knot semigroups are not a knot invariant.

A different example demonstrating this can be found in [10]. That example is more exotic because it uses split links, which are anomalous objects in knot theory ${ }^{5}$. The example given here is more mainstream. The difference between diagrams $K \mathfrak{t w}_{2}^{0}$ and $K \mathfrak{t w}_{1}^{1}$ is a half-turn known as a flype in knot theory; thus, flypes do not preserve knot semigroups.

On a more general note, this example shows that a knot semigroup expresses some properties of both the knot and the specific knot diagram it is built from. Thus, they may become a useful tool in research which requires comparing different knot diagrams of the same nnot $^{6}$.

\footnotetext{
${ }^{4}$ More generally, links $\mathfrak{t w}_{c}^{d}$ and $\mathfrak{t w}_{c+d}^{0}$ are equivalent for all values of $c$ and $d$.

${ }^{5}$ For example, the main theorem in [2] (Section 5.2) showing that quandles are knot invariants excludes split links from consideration.

${ }^{6}$ The first author is now involved in research in which the process of untangling a knot diagram of the trivial knot is facilitated by considering knot semigroups of certain tangle diagrams.
} 


\section{Dihedral semigroups}

Consider a regular $n$-sided polygon. It has $n$ axes of symmetry; denote them consecutively (for example, clockwise) by $A_{0}, \ldots, A_{n-1}$. Denote the reflection about the axis $A_{i}$ by $s_{i}$. The group generated by $s_{0}, \ldots, s_{n-1}$ is the dihedral group $D_{n}$.

Another algebra expressing symmetries of a regular $n$-sided polygon is a dihedral kei, also known as dihedral quandle. It is an algebra whose elements are the symmetry axes $A_{0}, \ldots, A_{n-1}$ and whose binary operation $A_{i} \triangleright A_{j}$ is the reflection of $A_{i}$ about $A_{j}$. Equivalently, it is an algebra with elements $\{0, \ldots, n-1\}$ and the operation $i \triangleright j=2 j-i$, where $2 j-i$ is calculated in $\mathbb{Z}_{n}$. Or, equivalently, it is a subset $s_{0}, \ldots, s_{n-1}$ of $D_{n}$ with the operation $s_{i} \triangleright s_{j}=s_{j} s_{i} s_{j}$.

Both dihedral groups and dihedral keis have been repeatedly used to express properties of knots. As to dihedral groups, the idea was first introduced in [5], page 161, and is most clearly expressed in Fact 1. As to dihedral keis, a good description of the constructions used can be found in [6]; for recent computational results, see $[3,4,8]$. The purpose of this section is to link alternating sum semigroups to dihedral groups.

Let $B \subseteq \mathbb{Z}_{n}$. Introduce a homomorphism $\delta$ from $B^{+}$to $D_{n}$ induced by the mapping $b \mapsto s_{b}$ for each $b \in B$. Say that two words $u, v \in B^{+}$are in relation $\rho$ if $u$ and $v$ have the same length and $\delta(u)=\delta(v)$. Obviously $\rho$ is a congruence on $B^{+}$. We shall denote the factor semigroup $B^{+} / \rho$ by $D_{n}(B)$ and call it a dihedral semigroup, by an analogy with dihedral groups and dihedral keis.

Theorem 15. $A S\left(\mathbb{Z}_{n}, B\right)$ is isomorphic (actually, equal) to $D_{n}(B)$.

Proof. The multiplication table of $D_{n}$ can be written out explicitly as $s_{i} s_{j}=r_{i-j}$, $r_{i} s_{j}=s_{i+j}, s_{i} r_{j}=s_{i-j}$ and $r_{i} r_{j}=r_{i+j}$, where elements $r_{i}$ are rotations, and $i-j$ and $i+j$ are calculated in $\mathbb{Z}_{n}$. Inspecting the first two types of equalities, we conclude that a word consisting of only reflections $s_{i_{1}} \ldots s_{i_{k}}$ is equal in $D_{n}$ to either $r_{\alpha}$, if $k$ is even, or $s_{\alpha}$, if $k$ is odd, where $\alpha$ is the alternating sum (calculated in $\mathbb{Z}_{n}$ ) of the word consisting of the indices $i_{1} \cdots i_{k}$. Thus, providing that two words $u, v \in B^{+}$have the same length, $\delta(u)=\delta(v)$ if and only if $\operatorname{alt}(u)=\operatorname{alt}(v)$. Therefore, $u$ and $v$ of an equal length are equal in $A S\left(\mathbb{Z}_{n}, B\right)$ if and only if they are equal in $D_{n}$ and, therefore, if and only if they are equal in $D_{n}(B)$. In other words, congruence $\rho$ defined above and congruence $\sim$ defined in Section 1 are equal. Therefore, $A S\left(\mathbb{Z}_{n}, B\right)=B^{+} / \sim=B^{+} / \rho=D_{n}(B)$.

The proof above concentrates around the fact that two words of the same length are equal in $A S\left(\mathbb{Z}_{n}, B\right)$ if and only if they are equal in $D_{n}$. Within the context of 4-plat diagrams (see Conjecture 2 and Fact 1), this can be interpreted as saying that two words of the same length are equal in $K \mathfrak{d}$ if and only if they are equal in $O \mathfrak{d}$. Generalising this further, one can ask if the following is true.

Conjecture 16. Providing that $\mathfrak{d}$ is a knot diagram, two words are equal in $K \mathfrak{d}$ if and only if they have the same length and are equal in $O \mathfrak{O}$.

If this result is true, it will help us to study knot semigroups of 4-plat diagrams (and prove Conjecture 2), since one will be able to refer to Fact 1 instead of proving complicated lemmas ${ }^{7}$ like in Section 3.

\footnotetext{
${ }^{7}$ Chapter 5 of [9] contains a useful discussion of how proofs in cancellative semigroups compare with those in groups.
} 
There is also another way of expressing a connection between alternating sum semigroups and symmetries of regular polygons, as the following observation shows (we do not include the proof, which is routine).

Proposition 17. The factor semigroup of $D_{n}(B)$ relative to the congruence induced by equalities $b b=1$ for each $b \in B$ is the dihedral group $D_{n}$.

From this observation it follows that Theorem 18 (for knot diagrams) is a corollary of Conjecture 2.

\section{Group Od of 4-Plat Diagrams}

The purpose of this section is to present a simple algebraic proof of the 'only if' part of Fact 1. To link this proof to other parts of the paper, it is useful to note that the proof employs canonical words $w_{i}$ which are very similar to words $w(m, i)$ in Section 2, the same numerical labels as in Section 3 and the same expressions at crossings as in dihedral keis in Section 4.

Theorem 18. For every 4-plat diagram $\mathfrak{d}$, group $O \mathfrak{d}$ is a dihedral group.

Proof. The proof will proceed by induction on the number of crossings in a braid, as the crossings of a 4-plat diagram are added one by one starting from the left, as the figure shows.
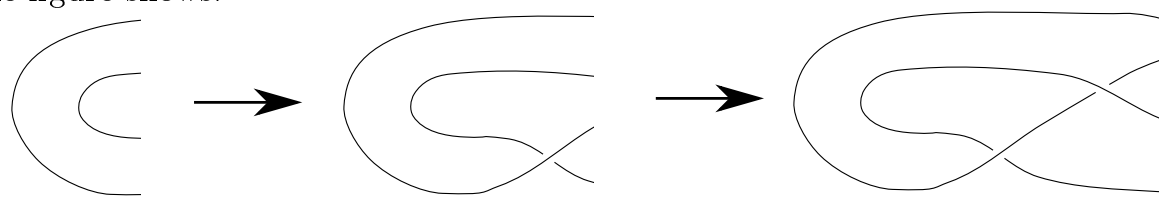

Let us introduce integer-valued labels for each arc. On the first diagram, let the label of the strand $x$ in position 1 be 0 , and let the label of the strand $y$ in position 3 be 1 . Whenever we 'grow the diagram to the right' by adding a new crossing, let the label of the newly formed arc on the right-hand side of the diagram be equal to $2 j-i$, where $i$ is the label of the arc terminating at the crossing and $j$ is the label of the arc passing over the crossing.

We shall call a quadruple of numbers $(i, j, p, q)$ a parallelogram if $i+p=j+q$. Let us prove that whenever we consider a left-hand side fragment of a 4-plat diagram, the labels of the four rightmost arcs (in the order of their positions, from the bottom to the top) form a parallelogram.

As to the base of the induction, the labels, considered from the bottom to the top, form a quadruple $(0,0,1,1)$ or $(0,1,1,0)$; these quadruples are parallelograms.

As to the step of the induction, consider a braid closed on the left in which the labels of the rightmost arcs are, from the bottom to the top, $(i, j, p, q)$, and $(i, j, p, q)$ is a parallelogram. Now suppose a new crossing is added; six different crossings are possible, and considering them all can be done in the same fashion; therefore, let us only consider one in detail, when the strand in position 1 crosses over the strand in position 2 (like the first crossing in the example in the figure). Then the label of the new arc in position 1 is $2 i-j$. The labels of the rightmost arcs are, from the bottom to the top, $(2 i-j, i, p, q)$. Then we have $(2 i-j)+p=(i+p)+i-j=$ $(j+q)+i-j=i+q$; hence, $(2 i-j, i, p, q)$ is a parallelogram.

Recall that $x$ and $y$ are the letters denoting the two left-most strands. For every positive integer $i$, let $w_{i}$ be the word $y(x y)^{i-1}$; for every non-positive integer $i$, let $w_{i}$ be the word $x(y x)^{-i}$. For a simpler notation, we do not distinguish between an 
arc and the corresponding element of $O \mathfrak{d}$. Let us prove that each $\operatorname{arc} a$ is equal in $O \mathfrak{d}$ to $w_{i}$, where $i$ is the label of $a$. Also, note that simultaneously we prove that the equality $a^{2}=1$ for each arc $a$ follows from $x^{2}=y^{2}=1$ : indeed, since $x^{2}=y^{2}=1$, we have $w_{i}^{2}=1$ for every word $w_{i}$; thus, if $a=w_{i}$ then $a^{2}=1$.

As to the base of the induction, $x=w_{0}$ and $y=w_{1}$.

As to the step of the induction, suppose a new crossing is added when an arc $b=w_{j}$ crosses over an $\operatorname{arc} a=w_{i}$. Then the label of the new $\operatorname{arc} c$ is $2 j-i$. At each crossing $a \dashv b \vdash c$, in $O \mathfrak{d}$ we have an equality $a b=c a$. Since $b=b^{-1}$, we have $c=b^{-1} a b=b a b=w_{j} w_{i} w_{j}$. It is routine to check that $w_{j} w_{i} w_{j}=w_{2 j-i}$; hence, $c=w_{2 j-i}$. (It is useful to stress that in this proof we treat the equality $c=b a b$ not as a defining relation of $O \mathfrak{d}$, but merely an indication that a certain element $w_{2 j-i} \in O \mathfrak{d}$ should be considered.)

After all crossings have been added, assume that the rightmost arcs have labels $(i, j, p, q)$. Then the braid is closed on the right in one of two ways, thus producing a pair of equalities in $O \mathfrak{d}$, which is either $w_{i}=w_{j}$ and $w_{p}=w_{q}$ or $w_{i}=w_{q}$ and $w_{j}=w_{p}$. Let us consider the former case in detail; the latter can be considered in the same fashion. Since $(i, j, p, q)$ is a parallelogram, the equalities $w_{i}=w_{j}$ and $w_{p}=w_{q}$ are not two distinct equalities, but the same equality, which can be rewritten as $(x y)^{n}=1$, where $n=i-j$ or, equivalently, $n=p-q$.

Overall, this means that $O \mathfrak{d}$ can be defined by just three defining relations, being $x^{2}=1, y^{2}=1$ and $(x y)^{n}=1$; all other equalities in $O \mathfrak{d}$ follow from these three. The group defined by such three defining relations is a dihedral group.

\section{REFERENCES}

[1] Michel Boileau and Bruno Zimmermann. The $\pi$-orbifold group of a link. Mathematische Zeitschrift, 200(2):187-208, 1989.

[2] Roger Fenn and Colin Rourke. Racks and links in codimension two. Journal of Knot theory and its Ramifications, 1(04):343-406, 1992.

[3] Andrew Fish and Alexei Lisitsa. Detecting unknots via equational reasoning, i: Exploration. In International Conference on Intelligent Computer Mathematics, pages 76-91. Springer, 2014.

[4] Andrew Fish, Alexei Lisitsa, and David Stanovský. A combinatorial approach to knot recognition. In Ross Horne, editor, Embracing Global Computing in Emerging Economies: First Workshop, EGC 2015, Almaty, Kazakhstan, February 26-28, 2015. Proceedings, pages 64-78. Springer International Publishing, 2015.

[5] R Fox. A quick trip through knot theory, in "topology of three-manifolds" ed. by mk fort, 1962.

[6] Seiichi Kamada. Knot invariants derived from quandles and racks. Geometry \& Topology Monographs, 4:4, 2002.

[7] Akio Kawauchi. A survey of knot theory. Birkhäuser, 1996.

[8] Alexei Lisitsa and Alexei Vernitski. Using automated reasoning to test hypotheses concerning knot semigroups. submitted.

[9] William McCune and Ranganathan Padmanabhan. Automated deduction in equational logic and cubic curves, volume 1095. Springer Science \& Business Media, 1996.

[10] Alexei Vernitski. Describing semigroups with defining relations of the form $\mathrm{xy}=\mathrm{yz}$ and $\mathrm{yx}=\mathrm{zy}$ and connections with knot theory. Semigroup Forum, pages 1-17, 2016. 
Department of Mathematical Sciences, University of Essex, Colchester, UK

E-mail address: asvern@essex.ac.uk

Mathematical Department, Tripoli University, Libya

E-mail address: laila.tunsi@gmail.com

INSA de Rouen, France

E-mail address: colette.ponchel@insa-rouen.fr

Department of Computer Science, University of Liverpool, Liverpool, UK

E-mail address: A.Lisitsa@liverpool.ac.uk 